\title{
On numerical methods for highly oscillatory problems in circuit simulation
}

\author{
Marissa Condon \\ School of Electronic Engineering \\ Dublin City University \\ Dublin 9 \\ Ireland \\ Alfredo Deaño \\ Department of Applied Mathematics and Theoretical Physics \\ Centre for Mathematical Sciences \\ University of Cambridge \\ Wilberforce Rd, Cambridge CB3 0WA, UK \\ Arieh Iserles \\ Department of Applied Mathematics and Theoretical Physics \\ Centre for Mathematical Sciences \\ University of Cambridge \\ Wilberforce Rd, Cambridge CB3 0WA, UK \\ Kornel Maczynski \\ School of Electronic Engineering \\ Dublin City University \\ Dublin 9 \\ Ireland \\ Tao Xu \\ School of Electronic Engineering \\ Dublin City University \\ Dublin 9 \\ Ireland
}

October 7, 2008

\begin{abstract}
We propose in this paper a novel technique for an efficient numerical approximation of systems of highly oscillatory ordinary differential equations. In particular, we consider electronic systems subject to modulated signals. A Filon-type method is proposed for use and compared with traditional trapezoidal rule and Runge-Kutta methods. The Filontype method is combined with the waveform relaxation technique for nonlinear systems. Preliminary numerical examples highlight the efficacy of this approach.
\end{abstract}




\section{Introduction}

Most numerical simulators of electric and electronic circuits, such as SPICE (Nagel 1975), as well as general-purpose solvers of ordinary differential equations (ODEs), like those in MATLAB, use either multistep or Runge-Kutta methods. This is perfectly adequate for a great majority of ODEs in applications, yet falls woefully short for systems subject to modulated signals or RF oscillators. In this setting, traditional quadrature approaches can necessitate the use of minute step-sizes with the consequent outcome of great inefficiencies and often impractical simulation times.

Modulation is the process whereby information is transmitted at a high frequency to enable antennae of practical dimensions to be employed. In amplitude modulation (AM), the information signal/envelope is in the form of a low frequency sinewave, $A_{\mathrm{m}} \sin \omega_{\mathrm{m}} t$. The resultant amplitude-modulated signal is therefore $A_{\mathrm{c}}\left(1+m \sin \omega_{\mathrm{m}} t\right) \sin \omega_{\mathrm{c}} t$, where $m=$ $A_{\mathrm{m}} / A_{\mathrm{c}}$. Another variation of amplitude modulation is the Double-Sided Suppressed Carrier $A_{\mathrm{m}} \sin \omega_{\mathrm{m}} t \sin \omega_{\mathrm{c}} t . \omega_{\mathrm{c}}=2 \pi f_{\mathrm{c}}$ is the carrier frequency in rad/s and $A_{\mathrm{c}}$ is its amplitude. In general AM the information signal is in the $\mathrm{KHz}$ range, while the carrier signal is in the $\mathrm{MHz}$ range.

In digital modulation the information to be transmitted is a sequence of ones and zeros, termed bits. The amplitude, frequency or phase of a carrier signal is varied dependent on the bit value. For example, in Binary Phase Shift Keying (BPSK) the modulated signal is of the form $b(t)=\cos \left(\omega_{\mathrm{c}} t\right) x(t)$, where $x(t)$ is $+A$ or $-A$ if a ' 1 ' or a ' 0 ' bit is to be transmitted, respectively. In digital technologies involving more complex formats, such as EDGE, the information/envelopes have bandwidths in the $\mathrm{KHz}$ range, while the carriers are $800 \mathrm{MHz}$ and $1800 \mathrm{MHz}$. For evaluation of a bit-error ratio of such an RF transmission, several information envelope time periods are required, but the step size is governed by the underlying carrier frequency which is significantly higher than the envelope frequency.

All this motivates the present contribution, which addresses the issue of simulating ODEs involving very high frequencies and widely varying frequency ranges. In particular, we intend to demonstrate that Filon-type methods, applied in tandem with exponential integrators, result in increased efficiencies for systems involving signal of widely varying frequencies. Similar techniques have been already investigated for different models of highly oscillatory ODEs by Khanamirian (2008).

Some of the results in this paper have been already addressed from the mathematical standpoint in (Condon, Deaño \& Iserles 2008).

\section{ODEs and RF systems}

The general solution of the ordinary differential equation

$$
\boldsymbol{y}^{\prime}=A \boldsymbol{y}+\boldsymbol{g}(t, \boldsymbol{y}), \quad t \geq 0, \quad \boldsymbol{y}(0)=\boldsymbol{y}_{0},
$$

can be written using the variation of constants formula in the following implicit form,

$$
\boldsymbol{y}(t)=\mathrm{e}^{t A} \boldsymbol{y}_{0}+\int_{0}^{t} \mathrm{e}^{A(t-\tau)} \boldsymbol{g}(\tau, \boldsymbol{y}(\tau)) \mathrm{d} \tau
$$

In the current paper, the focus is on a specific type of circuit, such as the diode rectifier shown in Fig. 2.1 and on ODEs (2.1) that occur in their modelling (Dautbegovic, Condon \& 
Brennan 2005). We have focused on such circuits dues to their highly nonlinear, yet simple nature. Two different input signals will be considered, an analogue AM-modulated signal and a digitally-modulated signal.

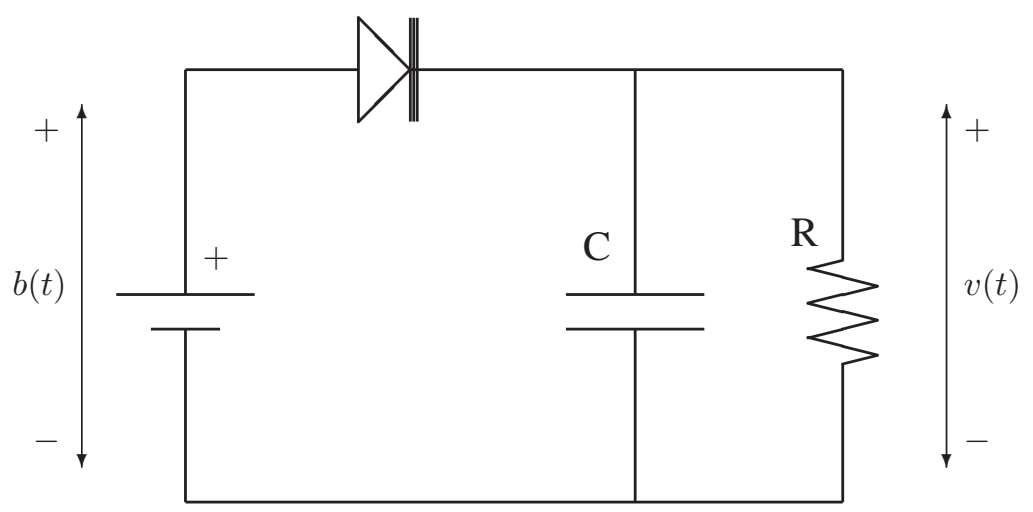

Figure 2.1: Diode rectifier circuit.

The governing equation for the circuit in Fig. 2.1 is

$$
I_{0}\left[\mathrm{e}^{k(b(t)-v(t))}-1\right]=C \frac{\mathrm{d} v(t)}{\mathrm{d} t}+\frac{v(t)}{R} .
$$

Here $C$ is capacitance, $R$ resistance, $I_{0}$ is a constant denoting the diode reverse bias current, $b(t)$ is the input signal and the unknown $v(t)$ is the voltage.

Once we apply the variations of constants formula (2.2) to the ODE (2.3), integrating from $t_{n}$ to $t_{n+1}=t_{n}+h$ rather than from the origin, we obtain

$$
\begin{aligned}
v_{n+1} & =v_{n} \mathrm{e}^{-h /(R C)}+R I_{0}\left[\mathrm{e}^{-h /(R C)}-1\right]+\frac{I_{0}}{C} \int_{t_{n}}^{t_{n+1}} \exp \left(\frac{t-t_{n+1}}{R C}-k v(t)+k b(t)\right) \mathrm{d} t \\
& =v_{n} \mathrm{e}^{-h /(R C)}+R I_{0}\left[\mathrm{e}^{-h /(R C)}-1\right]+\frac{I_{0}}{C} \mathcal{I}[g]
\end{aligned}
$$

where

$$
g(t)=\exp \left(\frac{t-t_{n+1}}{R C}-k v(t)+k b(t)\right) \quad \text { and } \quad \mathcal{I}[g]=\int_{t_{n}}^{t_{n+1}} g(t) \mathrm{d} t .
$$

If $b(t)=\varepsilon \cos \vartheta(t)$ then the integral in(2.4) is of the form

$$
\mathcal{I}[g]=\int_{t_{n}}^{t_{n+1}} f(t, v(t)) \mathrm{e}^{z \cos \vartheta(t)} \mathrm{d} t,
$$

where

$$
z=k \varepsilon, \quad f(t, v(t))=\exp \left[\frac{t-t_{n+1}}{R C}-k v(t)\right] .
$$

Integrals of similar form have been already investigated by Condon et al. (2008). 
It follows from (Abramowitz \& Stegun 1964, p. 376) that

$$
\begin{aligned}
\mathrm{e}^{z \sin \vartheta} & =\mathrm{I}_{0}(z)+2 \sum_{k=0}^{\infty}(-1)^{k} \mathrm{I}_{2 k+1}(z) \sin (2 k+1) \vartheta+2 \sum_{k=1}^{\infty}(-1)^{k} \mathrm{I}_{2 k}(z) \cos 2 k \vartheta, \\
\mathrm{e}^{z \cos \vartheta} & =\mathrm{I}_{0}(z)+2 \sum_{k=1}^{\infty} \mathrm{I}_{k}(z) \cos k \vartheta
\end{aligned}
$$

where $\mathrm{I}_{k}$ is the $k$ th modified Bessel function. Consequently, we can write the integral $I[g]$ in the form

$$
\begin{aligned}
I[g] & =\int_{t_{n}}^{t_{n+1}} f(t, v(t)) \mathrm{e}^{z \cos \vartheta(t)} \mathrm{d} t \\
& =\mathrm{I}_{0}(z) \int_{t_{n}}^{t_{n+1}} f(t, v(t))+2 \sum_{k=1}^{\infty} \mathrm{I}_{k}(z) \int_{t_{n}}^{t_{n+1}} f(t, v(t)) \cos (k \vartheta(t)) \mathrm{d} t,
\end{aligned}
$$

thereby expressing it as an infinite sum of integrals. The virtue of the highly oscillatory integrals on the right of (2.6) is that they are amenable to very rapid and efficient numerical calculation with Filon-type methods (Iserles \& Nørsett 2005).

Next we consider the more complicated case of analogue amplitude modulation, whereby $b(t)=\sin \left(\omega_{1} t\right) \sin \left(\omega_{2} t\right)$. The first step is to rewrite $b(t)$ in the form

$$
b(t)=\frac{1}{2}\left[\cos \left(\tilde{\omega}_{1} t\right)-\cos \left(\tilde{\omega}_{2} t\right)\right],
$$

where $\tilde{\omega}_{1}=\omega_{1}-\omega_{2}$ and $\tilde{\omega}_{2}=\omega_{1}+\omega_{2}$. This allows us to express the integral $I[g]$ as a product of two infinite series,

$$
\begin{aligned}
I[g]= & \int_{t_{n}}^{t_{n+1}} f(t, v(t)) \exp \left(\frac{1}{2} z \cos \tilde{\omega}_{1} t-\frac{1}{2} z \cos \tilde{\omega}_{2} t\right) \mathrm{d} t \\
= & {\left[I_{0}\left(\frac{1}{2} z\right)\right]^{2} \int_{t_{n}}^{t_{n+1}} f(t, v(t)) \mathrm{d} t+2 \mathrm{I}_{0}\left(\frac{1}{2} z\right) \sum_{k=1}^{\infty} \mathrm{I}_{k}\left(\frac{1}{2} z\right) \int_{t_{n}}^{t_{n+1}} f(t, v(t)) \cos \left(k \tilde{\omega}_{1} t\right) \mathrm{d} t } \\
& +2 \mathrm{I}_{0}\left(\frac{1}{2} z\right) \sum_{l=1}^{\infty}(-1)^{l} \mathrm{I}_{l}\left(\frac{1}{2} z\right) \int_{t_{n}}^{t_{n+1}} f(t, v(t)) \cos \left(l \tilde{\omega}_{2} t\right) \mathrm{d} t \\
& +4 \sum_{k=1}^{\infty} \sum_{l=1}^{\infty}(-1)^{l} \mathrm{I}_{k}\left(\frac{1}{2} z\right) \mathrm{I}_{l}\left(\frac{1}{2} z\right) \int_{t_{n}}^{t_{n+1}} f(t, v(t)) \cos \left(k \tilde{\omega}_{1} t\right) \cos \left(l \tilde{\omega}_{2} t\right) \mathrm{d} t .
\end{aligned}
$$

Since integrals with cosine oscillators can be calculated very efficiently using the methods of (Iserles \& Nørsett 2005), the above formula can be used as a means to calculate $I[g]$ to high accuracy. This is not totally straightforward, because for values of $k$ and $l$ for which $k \tilde{\omega}_{1} \pm l \tilde{\omega}_{2}$ vanishes (or is very small) the relevant integral in the double sum is non-oscillatory. This, in particular, means that, for rational $\tilde{\omega}_{1} \gg 1$ and $\tilde{\omega_{2}}$ it is true that

$$
\begin{aligned}
I[g] \sim\left[\mathrm{I}_{0}\left(\frac{1}{2} z\right)\right]^{2} \int_{t_{n}}^{t_{n+1}} f(t, v(t)) \mathrm{d} t+2 \sum_{\substack{k, l \geq 1 \\
k \tilde{\omega}_{1}+l \tilde{\omega}_{2}=0}}(-1)^{l} \mathrm{I}_{k}\left(\frac{1}{2} z\right) \mathrm{I}_{l}\left(\frac{1}{2} z\right) \int_{t_{n}}^{t_{n+1}} f(t, v(t)) \mathrm{d} t \\
+2 \sum_{\substack{k, l \geq 1 \\
k \tilde{\omega}_{1}=l \tilde{\omega}_{2}}}(-1)^{l} \mathrm{I}_{k}\left(\frac{1}{2} z\right) \mathrm{I}_{l}\left(\frac{1}{2} z\right) \int_{t_{n}}^{t_{n+1}} f(t, v(t)) \mathrm{d} t+\mathcal{O}\left(\omega^{-1}\right) .
\end{aligned}
$$


However, there is no need to use (2.7) for computational purposes. Insofar as we are concerned in this paper, the main importance of this asymptotic expansion is as a justification of the Filon-type methods of the next section.

\section{Filon-type methods and waveform relaxation}

Let

$$
\mathcal{F}[f]=\int_{a}^{b} f(t) G(t, \omega) \mathrm{d} t
$$

be a highly oscillatory integral: more specifically, the oscillator $G$ oscillates rapidly for $\omega \gg$ 1 , while $f$ itself is nonoscillatory. Typical examples of oscillators are $G(x, \omega)=\mathrm{e}^{\mathrm{i} \omega g(x)}$ and $G(x, \omega)=\mathrm{J}_{\nu}(\omega x)$, where $g$ is some given smooth function, while $\mathrm{J}_{\nu}$ is a Bessel function. The calculation of (3.1) for large $\omega$ by classical methods (e.g. Gaussian quadrature) is prohibitively expensive, but such integrals can be calculated with relative ease using recently developed methods, in particular Filon-type integrators (Iserles \& Nørsett 2005).

The idea underlying Filon-type methods, analysed and underpinned by much mathematical analysis in (Iserles \& Nørsett 2004, Iserles \& Nørsett 2005) and elsewhere, is to replace the function $f$ in (3.1) by a polynomial interpolation. Specifically, let

$$
a=c_{1}<c_{2}<\cdots<c_{q}=b
$$

be given nodes, each $c_{k}$ equipped with multiplicity $m_{k} \geq 1$. We construct a polynomial $p$ of degree $r=\sum_{k=1}^{q} m_{k}-1$ such that

$$
p^{(j)}\left(c_{k}\right)=f^{(j)}\left(c_{k}\right), \quad j=0,1, \ldots, m_{k}-1, \quad k=1,2, \ldots, \nu .
$$

The Filon-type method for the integral (3.1) is

$$
Q_{\mathrm{F}}[f]=\int_{a}^{b} p(t) G(t, \omega) \mathrm{d} t
$$

and it is premised upon the assumption that the integral (3.1) can be calculated explicitly for polynomial function $f$.

The interpolating polynomial $p$ can be written explicitly in the form

$$
p(t)=\sum_{k=1}^{q} \sum_{j=0}^{m_{k}-1} \alpha_{k, j}(t) f^{(j)}\left(c_{k}\right)
$$

where each $\alpha_{k, j}$ is itself a polynomial: as a matter of fact, it is the cardinal polynomial of Hermite interpolation, $\alpha_{k, j}^{(j)}\left(c_{k}\right)=1$, otherwise $\alpha_{k, j}^{(i)}\left(c_{l}\right)=0$ for all $i=0,1, \ldots, m_{l}-1$, $l=1,2, \ldots, q$. Therefore (3.2) can be written in the form

$$
Q_{\mathrm{F}}[f]=\sum_{k=1}^{q} \sum_{j=0}^{m_{k}-1} b_{k, j}(\omega) f^{(j)}\left(c_{k}\right), \quad \text { where } \quad b_{k, j}(\omega)=\int_{a}^{b} \alpha_{k, j}(t) G(t, \omega) \mathrm{d} t .
$$

The reason why Filon-type methods are so efficient is that suitable choice of nodes and multiplicities results in an exceedingly rapid decay of the error when $\omega \gg 1$. (The justification 
for this is provided by an asymptotic expansion: this is why (2.7) is so critical to our work.) For example, let us consider $G(x, \omega)=\mathrm{e}^{\mathrm{i} g(x)}$. If $g^{\prime} \neq 0$ in $[a, b]$ then $Q_{\mathrm{F}}[f]-\mathcal{F}[f] \sim$ $\mathcal{O}\left(\omega^{-s-1}\right), \omega \gg 1$, where $s=\min \left\{m_{1}, m_{q}\right\}$. In the case when $g^{\prime}$ vanishes in $[a, b]$, we must choose each such stationary point as a node of suitable multiplicity, and this ensures asymptotic decay of the error as $\mathcal{O}\left(\omega^{-\kappa}\right)$ for suitable number $\kappa$ (Iserles \& Nørsett 2005). In general, Filon-type methods possess the intriguing (and most welcome) feature that their precision increases as frequency of oscillation grows!

The Filon-type method corresponding to (2.6) is

$$
\mathcal{Q}_{\mathrm{F}}[g]=\mathrm{I}_{0}(z) \int_{t_{n}}^{t_{n+1}} p(t) \mathrm{d} t+2 \sum_{k=1}^{\infty} \mathrm{I}_{k}(z) \int_{t_{n}}^{t_{n+1}} p(t) \cos (k \vartheta(t)) \mathrm{d} t
$$

where $p(t)$ interpolates the function $f(t, v(t))$.

The infinite series in (3.3) converges very rapidly: since $\mathrm{I}_{k}(z)=\mathrm{i}^{-k} \mathrm{~J}_{k}(\mathrm{i} z)$ and $\mathrm{J}_{k}(z) \sim$ $\left(\frac{\mathrm{e} z}{2 k}\right)^{k} / \sqrt{2 \pi k}$ (Abramowitz \& Stegun 1964, p. 365), it is true that

$$
\mathrm{I}_{k}(z) \sim \frac{1}{\sqrt{2 \pi k}}\left(\frac{\mathrm{e} z}{2 k}\right)^{k}, \quad z \in \mathbb{R}, \quad k \gg 1,
$$

a hyper-exponential decay. Therefore, we can replace (3.3) by

$$
\mathcal{Q}_{\mathrm{F}}[g]=\mathrm{I}_{0}(z) \int_{t_{n}}^{t_{n+1}} p(t) \mathrm{d} t+2 \sum_{k=1}^{N} \mathrm{I}_{k}(z) \int_{t_{n}}^{t_{n+1}} p(t) \cos (k \vartheta(t)) \mathrm{d} t
$$

for a relatively small value of $N$ without any ill effect insofar as accuracy is concerned.

Greater challenge is presented by the fact that the unknown $v(t)$ features inside the integral sign - the ODE being nonlinear, the variation of constants formula (2.4) is implicit. To this end we use waveform relaxation (WR). Several waveform relaxation methods have been developed in the last few decades, see for example (Miekkala \& Nevanlinna 1996, Nevanlinna 1989, Vandewalle 1993).

The standard form of WR for an ODE of the form (2.1) is

$$
\begin{aligned}
& \boldsymbol{y}^{[0]}(t) \equiv \boldsymbol{y}_{0}, \\
& \boldsymbol{y}^{[s]}(t)=\mathrm{e}^{t A} \boldsymbol{y}_{0}+\int_{0}^{t} \mathrm{e}^{(t-\tau) A} \boldsymbol{g}\left(\tau, \boldsymbol{y}^{[s-1]}(\tau)\right) \mathrm{d} \tau, \quad s=1,2, \ldots,
\end{aligned}
$$

and this can be easily generalized to a time-stepping algorithm from $t_{n}$ to $t_{n+1}$.

Combining the simplest Filon-type quadrature with (3.4) results in the following iterative 
scheme, that needs to be executed within each time step.

$$
\begin{aligned}
v_{n+1}^{[0]}= & v_{n} \\
& \tilde{v}^{[0]}(t)=\frac{t_{n+1}-t}{h} v_{n}+\frac{t-t_{n}}{h} v_{n+1}^{[0]}\left(\equiv v_{n}\right), \\
v_{n+1}^{[1]}= & v_{n} \mathrm{e}^{-h /(R C)}+R I_{0}\left[\mathrm{e}^{-h /(R C)}-1\right]+\frac{I_{0}}{C} \int_{t_{n}}^{t_{n+1}} \mathrm{e}^{k b(t)} p\left(t, \tilde{v}^{[0]}(t)\right) \mathrm{d} t, \\
& \tilde{v}^{[1]}(t)=\frac{t_{n+1}-t}{h} v_{n}+\frac{t-t_{n}}{h} v_{n+1}^{[1]}, \\
v_{n+1}^{[2]}= & v_{n} \mathrm{e}^{-h /(R C)}+R I_{0}\left[\mathrm{e}^{-h /(R C)}-1\right]+\frac{I_{0}}{C} \int_{t_{n}}^{t_{n+1}} \mathrm{e}^{k b(t)} p\left(t, \tilde{v}^{[1]}(t)\right) \mathrm{d} t, \\
& \tilde{v}^{[2]}(t)=\frac{t_{n+1}-t}{h} v_{n}+\frac{t-t_{n}}{h} v_{n+1}^{[2]},
\end{aligned}
$$

Thus, the Filon nodes are $c_{1}=t_{n}$ and $c_{2}=t_{n+1}$, with unit multiplicities at both points: the linear polynomial $p$ agrees with $f\left(t, \tilde{v}^{[i]}(t)\right.$ at $t=t_{n}$ and $t_{n+1}$. The iteration is terminated once $\left|v_{n+1}^{[r]}-v_{n+1}^{[r-1]}\right|<$ TOL, whence we let $v_{n+1}=v_{n+1}^{[r]}$.

The choice of just two nodes is essential, because the inclusion of interior points would have greatly complicated the algorithm. To increase the precision of the algorithm we would have needed to interpolate to both $f(t, v(t))$ and

$$
\frac{\mathrm{d}}{\mathrm{d} t} f(t, v(t))=\frac{\partial f(t, v(t))}{\partial t}+\frac{\partial f(t, v(t))}{\partial v} v^{\prime}(t)
$$

at the endpoints. (Note that $v^{\prime}$ can be obtained from $v$ using the differential equation.) The purpose of this paper being an introduction of a new concept, rather than of a fully-fledged, tested and optimized algorithm, we dwell no more on that issue and restrict ourselves to linear interpolation.

\section{Numerical results}

\subsection{Amplitude modulation}

We commence from the case where $b(t)=\sin \omega_{1} t \sin \omega_{2} t$, with $\omega_{1}=100 \mathrm{rad} / \mathrm{s}$ and $\omega_{2}=10^{7}$ $\mathrm{rad} / \mathrm{s}$, difference of five orders of magnitude between the two oscillation scales. We prescribe $I_{0}=1, C=1, R=1$ and $k=40$ in Fig. 2.1 and equation (2.3).

Figure 4.1 depicts the exact solution of the ODE - in reality, a numerical solution with an exceedingly small step size. It is clear that, at the scale of the plot, the solution is a slow-varying wave and the extraordinarily large frequency $\omega_{2}$ is not visible. Nonetheless, the presence of high oscillation is enough to render traditional numerical methods inefficient. In Figs. 4.2-4 we exhibit the outcome of integrating the ODE in the interval $[0,0.2]$ with the constant step size $h=2.5 \times 10^{-4}$ and three numerical methods. In each case the numerical solution is denoted by solid line while, for comparison's sake, the exact solution from Fig. 4.1 features as a dashed line. 


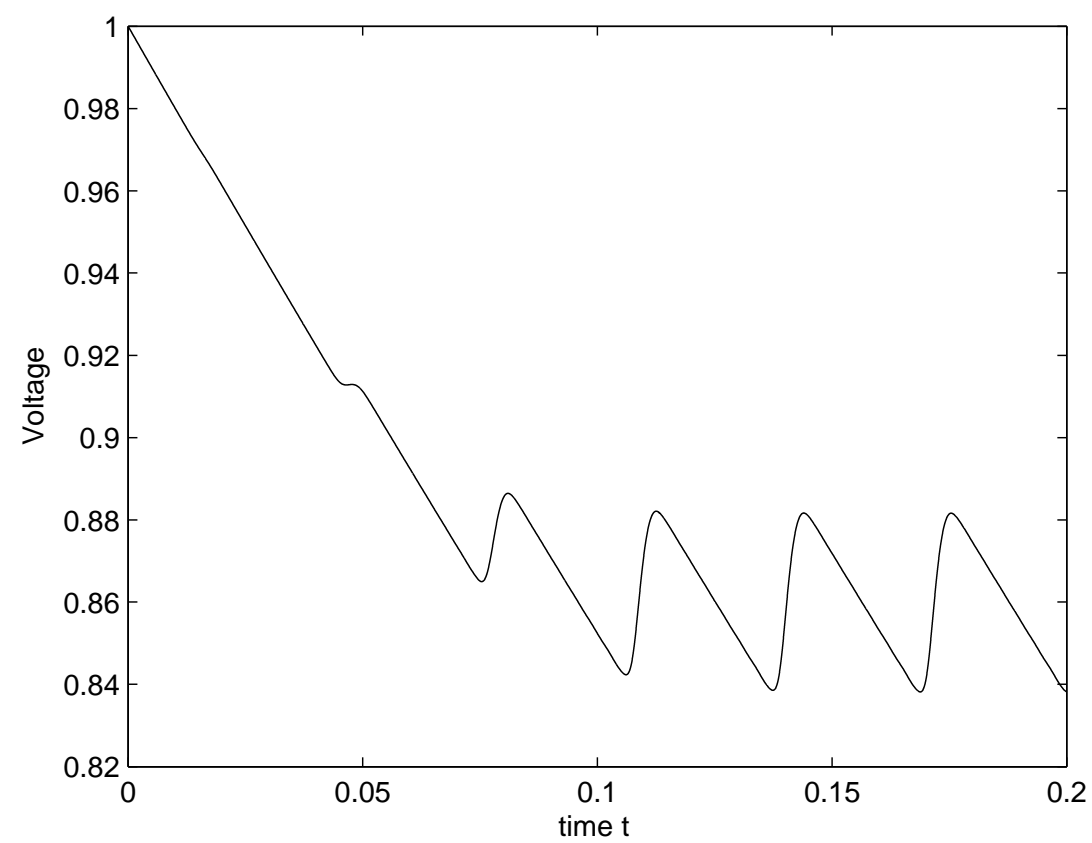

Figure 4.1: The exact solution of the amplitude-modulated equation with $\omega_{1}=100, \omega_{2}=$ $10^{7}, I_{0}=C=R=1$ and $k=40$.

The standard numerical mechanism to increase accuracy is to use a higher-order method, and this is evident from Figs. 4.2 and 4.3. The trapezoidal rule is of order 2, while the RungeKutta method that we used is of order 3 . The improvement in accuracy is tangible, but the main conclusion from both figures is that both methods deliver exceedingly poor results.

In comparison, the 'plain-vanilla' Filon-type method from Fig. 4.4, using just linear interpolation, produces a result which is visually hardly distinguishable from the exact solution. It is important to realise that the method is not designed to conform to the desiderata of classical numerical ODE solvers: both order and stability are meaningless in this context. Its efficacy comes from its good rendering of the asymptotics of highly oscillatory components.

Note further that our numerical examples are all using (the same) constant step and they forego the usual best practice of locally varying step size in response to an error control mechanism (Iserles 2008). This, we hasten to say, is not simply the consequence of employing rough-and-ready numerics on a toy example, in a way of a feasibility study. Modern allpurpose ODE software, with error-control and step-varying mechanism, performs exceedingly poorly in the presence of highly oscillatory components: it reduces the step size to an extent that renders the solution exceedingly expensive, while falling well short of attaining requested user tolerance (Condon et al. 2008, Iserles 2002). Ultimately, we expect Filon-type methods to be implemented with error control and variable step size, but at the present stage our intention is to demonstrate their superiority in comparison with classical solvers, and this point (we 


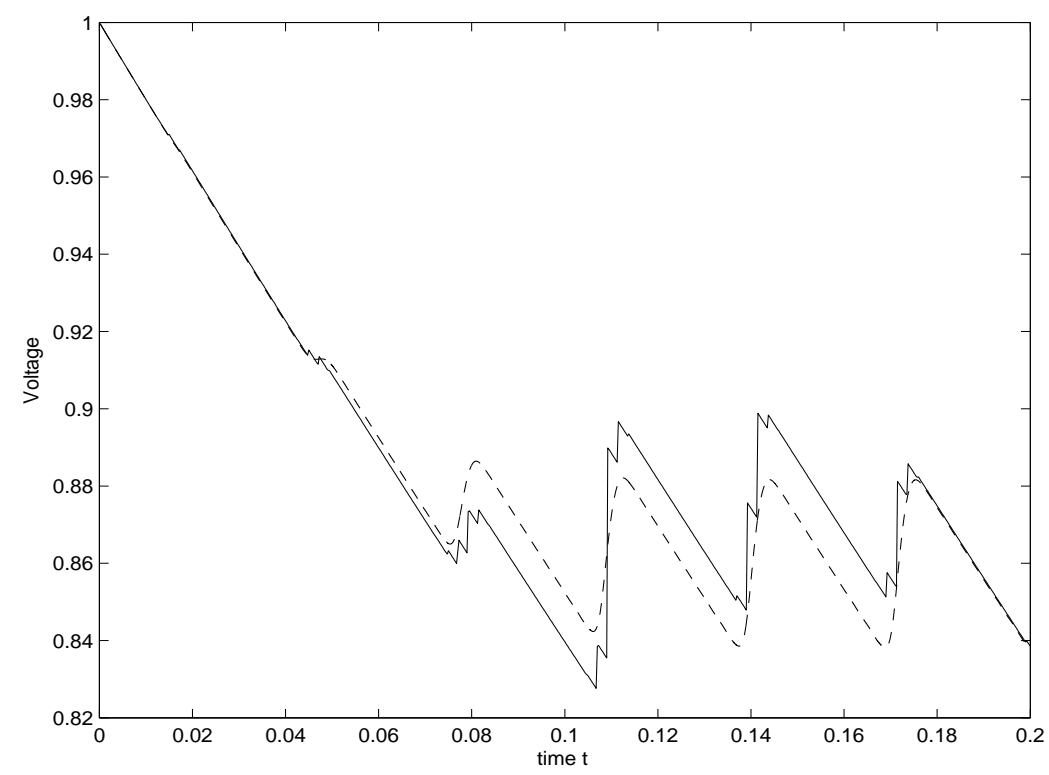

Figure 4.2: The solution of the amplitude-modulated equation by the trapezoidal rule.

believe) is amply made in Figs. 4.2-5.

As an aside, we comment that waveform relaxation converged fairly rapidly and letting $N=7$ our simulations (without even bothering to check for convergence) produced perfectly satisfactory results.

\subsection{Digital modulation}

As an example of digital modulation we have taken $b(t)=x(t) \cos \omega t$, where $\omega=4 \pi \times 10^{9}$ $\mathrm{rad} / \mathrm{s}$, while $x(t)$ is an alternating sequence of +1 and -1 with a bit period of $133 \mathrm{~ns} .{ }^{1}$ This is an example for the Binary Phase Shift Keying modulation technique. We let $I_{0}=100$, $C=10^{-4}, R=1$ and $k=1$.

The exact solution of the digitally-modulated equation with the above parameters is displayed in Fig. 4.5. The time scale itself is very short, because (as evident from the plot on the left) the rate of change is very substantial even for minute times. Equally important for efficient simulation is that observation, evident from the figure on the right (which zooms on an even smaller time-window), that the solution exhibits exceedingly rapid, small-amplitude oscillations. Such oscillations are invisible in less detailed plots but, nonetheless, are bound to defeat traditional ODE solvers or further depress their step size.

In Fig. 4.6 we display the errors committed by three numerical methods, all with constant step size $h=2.5 \times 10^{-10}$, applied to the digital-modulation equation: the (second-order)

\footnotetext{
${ }^{1}$ In a realistic model $x(t)$ is random, rather than alternating. However, introducing stochastic component would have made the comparison of different methods considerably more difficult.
} 


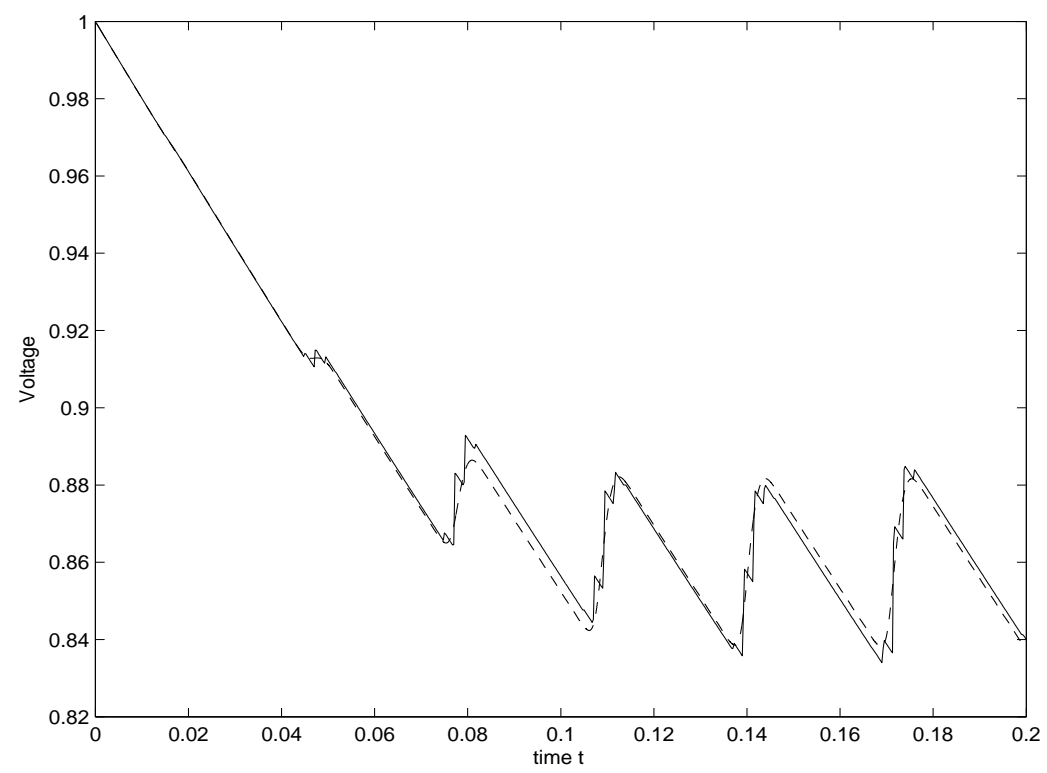

Figure 4.3: The solution of the amplitude-modulated equation by third-order explicit RungeKutta method.

trapezoidal rule, the standard explicit, third-order three-stage Runge-Kutta method and the Filon-type method with piecewise-linear approximation and waveform relaxation. The reason for the choice of the minute step size, roughly of the order of magnitude of $\mathcal{O}\left(\omega^{-1}\right)$, is that traditional methods require it, hence this course of action was unavoidable in this kind of comparison. Even taking such a tiny step size, it is evident how the numerical solution (solid line) rapidly departs from the exact solution, denoted by a dashed line. All accuracy is lost in even such a short interval! In comparison, the Filon-type method produces an outcome visually indistinguishable from the exact solution. Of course, given the way we constructed the Filon-type method, this remarkable feature survives if we deploy substantially larger step size: in methods designed using asymptotic principles the size of the step plays a minor role insofar as accuracy is concerned.

\section{Conclusions}

This paper presents a preliminary study into an alternative technique for numerical integration suitable for systems subjected to high-frequency signals. Numerical examples confirm the theoretical expectations on the significant potential of Filon-type methods in this setting.

Needless to say, this paper lays no claims to a comprehensive treatment of the subject, whether from mathematical, electronic engineering or software engineering point of view, being merely an introduction to the subject and a feasibility study of preliminary ideas. It expands on the theoretical insight gained from (Condon et al. 2008) and we expect it to be 


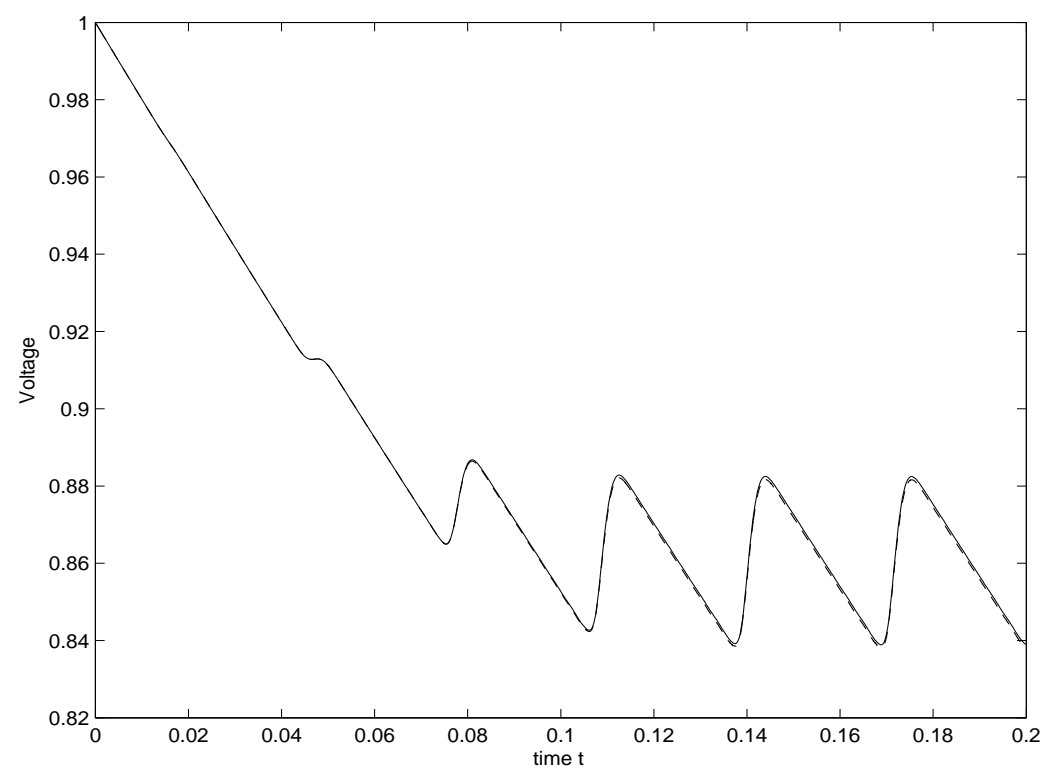

Figure 4.4: The solution of the amplitude-modulated equation by the simplest Filon-type method, combined with waveform relaxation.

followed by further publications, addressing this important subject with greater degree of detail.

\section{Acknowledgments}

The work of Marissa Condon, Kornel Maczynski and Tao Xu was supported by Science Foundation Ireland under Principal Investigator Grant No. 05/IN.1/I18, while Alfredo Deaño acknowledges financial support from the programme of postdoctoral grants of the Spanish Ministry of Education and Science.

\section{References}

Abramowitz, M. \& Stegun, I. A., eds (1964), Handbook of Mathematical Functions, National Bureau of Standards, Washington, DC.

Condon, M., Deaño, A. \& Iserles, A. (2008), On highly oscillatory problems arising in electronic engineering, Technical Report NA2008/10, DAMTP, University of Cambridge.

Dautbegovic, E., Condon, M. \& Brennan, C. (2005), 'An efficient nonlinear circuit simulation technique', IEEE Trans. Microwave Theory \& Techniques 53, 548-555. 

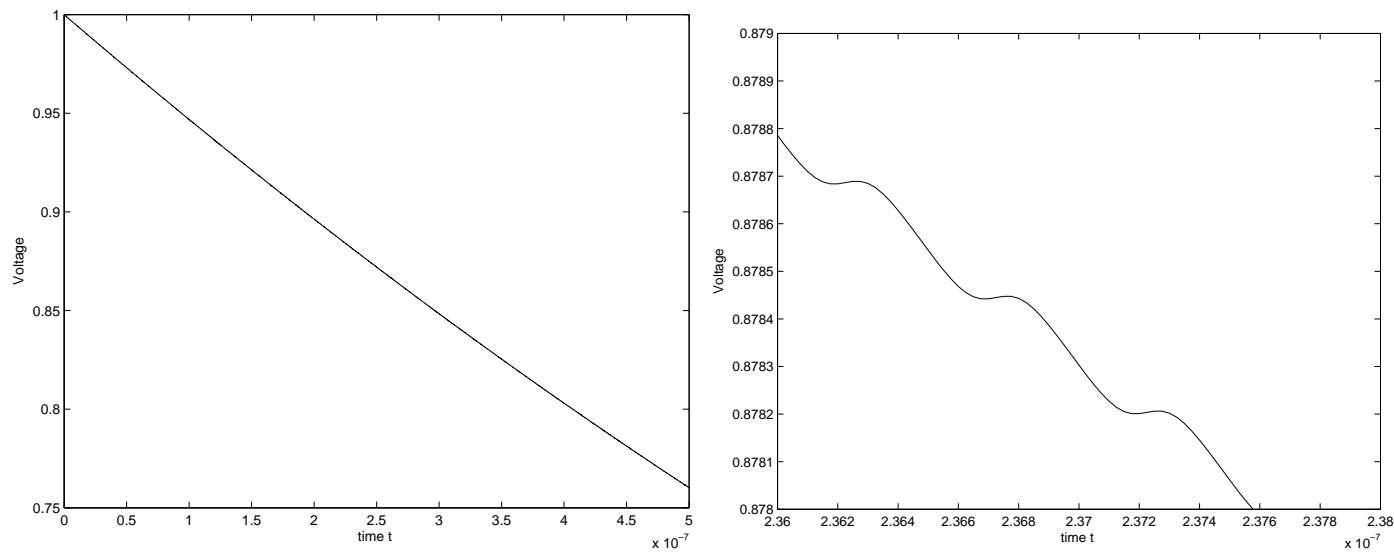

Figure 4.5: The exact solution of the digitally modulated equation. The plot on the right zooms into a very small time sub-interval.

Iserles, A. (2002), 'Think globally, act locally: solving highly-oscillatory ordinary differential equations', Appld Num. Anal. 43, 145-160.

Iserles, A. (2008), A First Course in the Numerical Analysis of Differential Equations, 2nd edn, Cambridge University Press, Cambridge.

Iserles, A. \& Nørsett, S. P. (2004), 'On quadrature methods for highly oscillatory integrals and their implementation', BIT 44, 755-772.

Iserles, A. \& Nørsett, S. P. (2005), 'Efficient quadrature of highly oscillatory integrals using derivatives', Proc. Royal Soc. A 461, 1383-1399.

Khanamirian, M. (2008), Quadrature methods for systems of highly oscillatory ODEs. part I, Technical Report NA2008/01, DAMTP, University of Cambridge.

Miekkala, U. \& Nevanlinna, O. (1996), 'Iterative solution of systems of linear differential equations', Acta Numerica 5, 259-307.

Nagel, L. W. (1975), Spice2, A computer program to simulate semiconductor circuits, Technical Report ERL-M520, Electrical Engineering, University of California, Berkeley.

Nevanlinna, O. (1989), 'Remarks on Picard-Lindelöff iteration', BIT 29, 328-346.

Vandewalle, S. (1993), Parallel multigrid waveform relaxation for parabolic problems, Teubner, Stuttgart. 

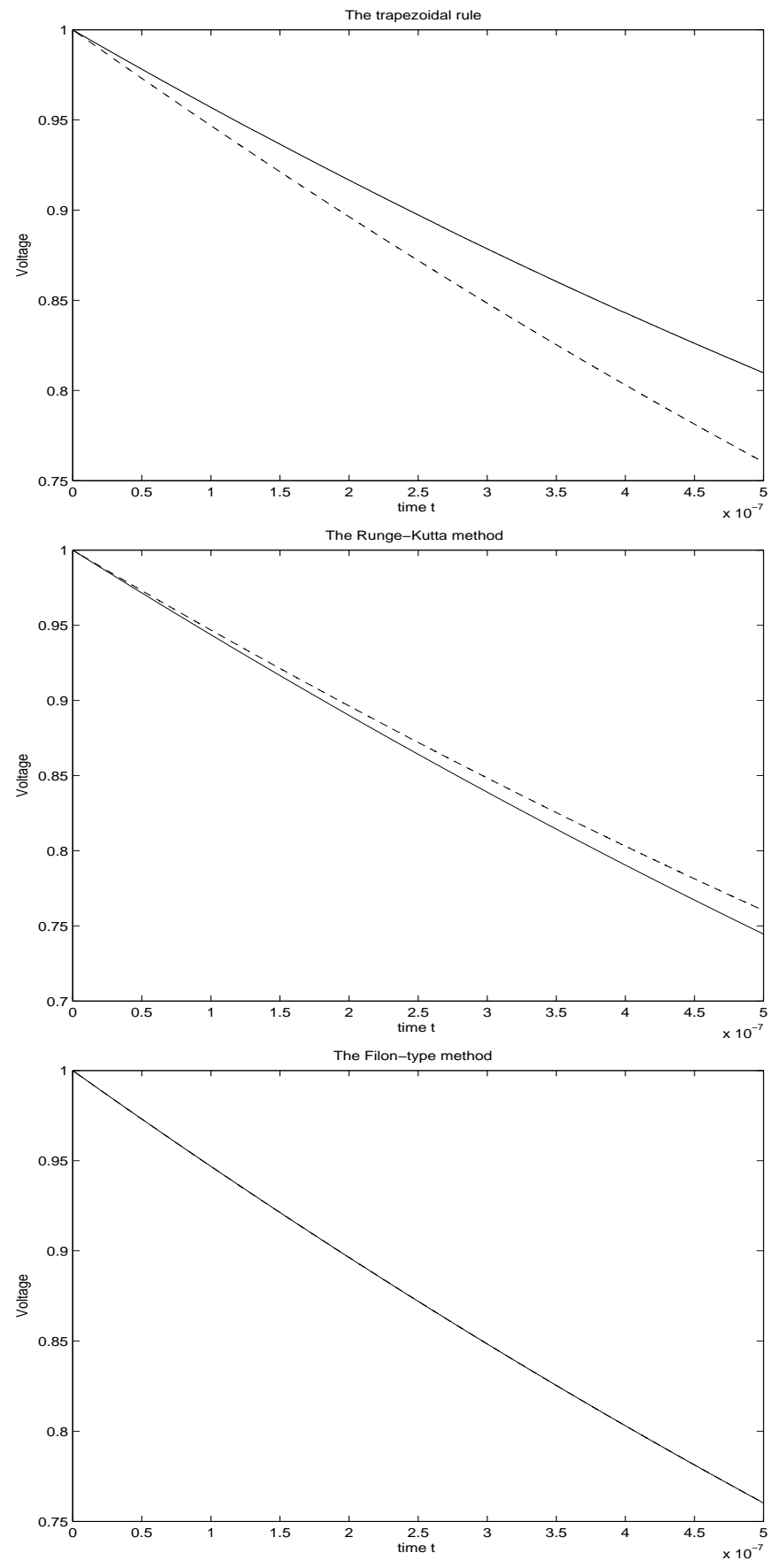

Figure 4.6: Numerical solution of the digitally-modulated equation by (a) the trapezoidal rule, (b) Runge-Kutta and (c) Filon-type method. 